\title{
Geometrical effects on radio pulsar profiles and spectra
}

\author{
Wolfgang Sieber \\ Fachhochschule Niederrhein, PF 2850, D-47728 Krefeld, Germany
}

\begin{abstract}
Considering the geometry of the hollow-cone model of radio pulsar emission in detail, one is led to the conclusion that several well-known observed effects may be explained quite easily purely on geometrical terms. It is shown that a steepening of the spectrum (cut-off) may be expected for certain geometrical situations as well as variations in component spectra, e.g. steeper spectra for the central component. These effects appear in addition to the already existing physical variations with frequency.
\end{abstract}

\section{Introduction}

The hollow-cone model (Radhakrishnan \& Cooke 1969, Ruderman \& Sutherland 1975) may be considered as a widely accepted working model for the emission geometry of radio pulsars. The model assumes in its basic form that the cone is centred on the magnetic field axis, pointing at latitude $\mu$ in a reference frame defined by the rotational axis of the neutron star. The radial extent of the cone may be given by the angle $\theta$. The chance orientation of the pulsar's rotation axis with respect to the line of sight to the observer determines the so-called "impact angle" $\beta$, i.e. the closest angular distance between the line of sight and the magnetic field axis. The (average) intensity distribution along the cut of the line of sight (through part of the hollow cone) determines in the end the observed average pulse profile.

The model predicts equal intensity for the leading and trailing half of the cone. Observations of many pulsars show however, that completely unpredictable variations of intensity occur across the profile. We will just disregard these intensity variations, since no theoretical explanation exists so far.

It should be noted, that it is well possible, that we miss important parts of the cone emission for those pulsars, where the cut of the line of sight is accidentally far off from the magnetic field axis.

\section{Best information from "central cuts" $\left(\beta \approx 0^{\circ}\right)$}

The best information about the structure of the emitting cone may be obtained from nearly, central cuts $\left(\beta \approx 0^{\circ}\right)$ (see also the more general discussion in Sieber 1996), because in this special geometry the intensity distribution across the cone and the width of the cone can be measured directly (albeit not the true width, for which the angle $\mu$ must be known). 
There are several investigations of this kind published (Thorsett 1991, Sieber et al. 1975, Phillips \& Wolszczan 1992) which show, that there exists a frequency dependence of the cone width in the sense, that the width increases at low frequencies (also called frequency to radius mapping). The separation $\Delta s$ between the two components may be described as:

$$
\Delta s=k \cdot f^{-\alpha}+\Delta s_{0}
$$

with $\alpha$ in the range $0.1 \leq \alpha \leq 0.3$

This frequency dependence of the observed (apparent) cone opening angle is a clearly measured effect, which seems to be an intrinsic property of the emission hollow-cone. We will in the following assume, that this property is preserved also for those pulsars, where not a clear double structure of the profile is visible due to the chance position of the line of sight cutting through the cone.

\section{Spectrum}

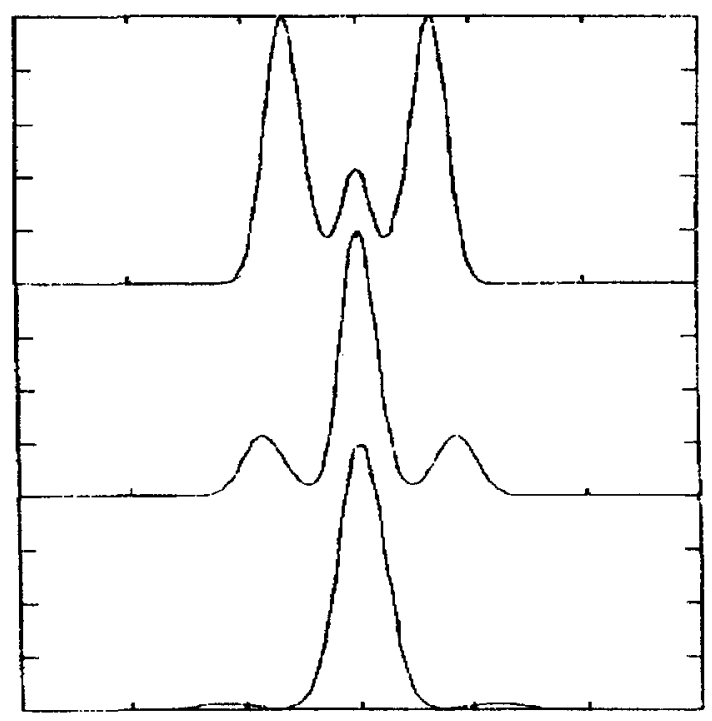

Figure 1. Frequency dependence of the simulated profile. From top to bottom: $2 \mathrm{GHz}, 800 \mathrm{MHz}, 200 \mathrm{MHz}$

The shrinking of the emission beam (hollow cone) at high frequencies may influence also the received intensity, i.e. the spectrum, of single components or of the whole profile. This may be demonstrated by simulating cuts through an artificial beam and by computing the resulting intensity.

Our artifical beam may consist of a gaussian beam centred on the magnetic field axis, the cone components (ring emission) may be given by a shifted gaussian beam resulting in profiles as shown in Fig. 1 in dependence of frequency. The core $I_{c}$, cone $I_{r}$ and total intensity

$$
I_{\text {tot }}=I_{c}+I_{r}
$$


is presented in Fig. 2.

It is obvious that the spectrum of the whole profile steepens considerably compared to the assumed intrinsic exponent for core and cone emission of -1.5 : We computed on average an observed exponent of -2.5 . This means that the "burden" on the physical radiation mechanism to produce steep spectra $(<-2)$ may be - at least for such geometrical arrangements - reduced considerably. It seems sufficient to have intrinsic spectral indices around -1.5 ; geometrical projection effects can then produce a steepening up to the observed values around $\leq-2$ (Sieber 1973).

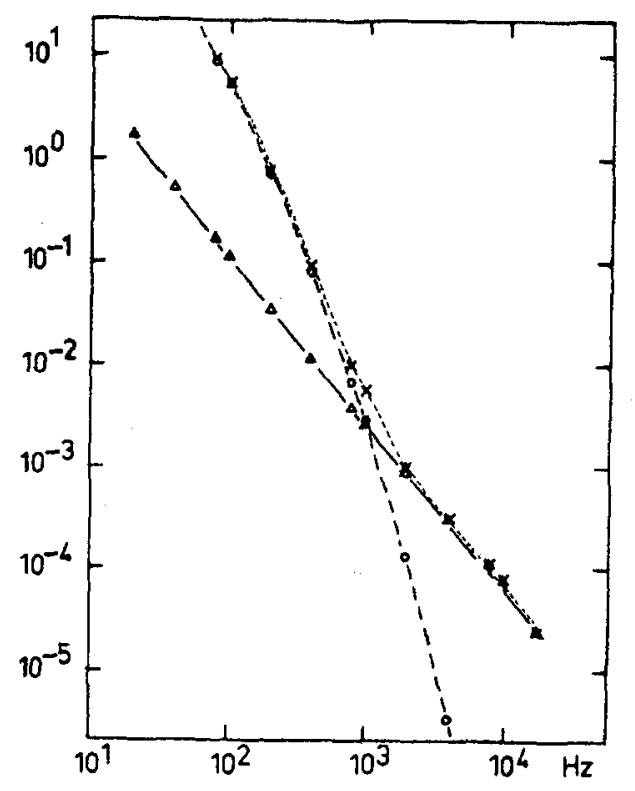

Figure 2. Spectrum of the simulated profile: Circles - core component, triangles - cone component, crosses - total intensity

Fig. 2 shows in addition, that a much steeper spectrum (compared to the rest of the profile) is observed for the core component, reaching (for the values used here) a minimum spectral index of -4.5 (purely due to geometrical projection effects). It is therefore in these cases not necessary to assume, that core component and cone component are produced by different physical mechanisms to explain the different spectral behaviour. It is interesting to note, that more or less the same conclusions have been derived independently by Kramer et al. (1994), who fitted the individual components of integrated profiles by gaussian profiles.

The figure suggests furthermore that the whole spectrum may steepen to high frequencies - as is observed in Fig. 2 for the core component alone - when no cone emission, or additional ring emission, exists. This effect might give an explanation for the so-called "cut-off" found in the spectra of many pulsars. 


\section{Conclusions}

Considering the geometry of the hollow-cone model in detail one is led to the following conclusions concerning mean pulse profile shapes and spectra.

From nearly central cuts one deduces that:

- The width $\theta$ of the cone is moderately frequency dependent resulting in narrower component separation and observed profile widths $\Delta w$ at higher frequencies.

Pulsars with grazing cuts can show additional features purely due to geometrical projection effects:

- A steepening of the overall spectrum (cut-off?),

- a steepening of the spectra of all components,

- steeper spectra for the core component compared to cone emission.

\section{References}

Kramer M., Wielebinski R., Jessner A., Gil J.A., Seiradakis J.H. 1994, A\&AS, 107,515

Phillips J.A., Wolszczan A. 1992, ApJ, 385, 273

Radhakrishnan V., Cooke D.J. 1969, Astrophys. Lett., 3, 225

Ruderman M.A., Sutherland P.G. 1975, ApJ, 196, 51

Sieber W. 1973, A\&A, 28, 237

Sieber W. 1996, A\&A, in press

Sieber W., Reinecke R., Wielebinski R. 1975, A\&A, 38, 169

Thorsett S.E. 1991, ApJ, 377, 263 